\title{
The Efficacy of Interaction Behavior and Internal Stiffness Control for Embodied Information Gain in Haptic Perception
}

\author{
Nantachai Sornkarn and Thrishantha Nanayakkara
}

\begin{abstract}
Haptic perception in biological systems not only depends on the environmental conditions, but also on the behavioral state and the internal impedance of the embodiment because proprioceptive sensors are embedded in the muscle and tendons used for actuation. A simple example of such a phenomenon can be found when people are asked to palpate a soft tissue to identify a stiff-inclusion. People tend to perform a variety of palpation strategies depending on their previous knowledge and the desired information. Does this mean that the probing behavioral variables and internal muscle impedance parameters and their interaction with given environmental conditions play a role in the perception information gain during the estimation of soft tissue's properties? In this paper, we use a two-degree of freedom laboratory-made variable stiffness and indentation probe to investigate how the modulation of probing behavioral and internal stiffness variables can affect the accuracy of the depth estimation of stiff inclusions in artificial silicon phantom tissue using information gain metrics based on prior knowledge in form of memory primitives.
\end{abstract}

\section{INTRODUCTION}

In an open surgery, a surgeon has direct access to targeted patient's tissues to perform direct diagnosis and desirable surgical activities with minimum constraint in the operations, which provides surgeons with a sense of touch at the finger tips to perceive the physical properties of the tissues. However, this method suffers from the long recovery time and pain caused to the patients. Therefore, in the past decades, different surgical operations, if permitted, are performed using a 'Minimally Invasive Surgery' (MIS) technique, which involves the insertion of tools through small trocar ports around the diagnosed area. As opposed to the traditional method, the surgeon only receives visual feedback of the environment through an endoscopic camera in an MIS. While this suffices to a certain extent in some surgical activities; it is desirable for the surgeon to receive a sense of touch in MIS [1]. A final verification of surgical site is essential before any surgical site decision despite the availability of the MRI images, as the tissue can still move due to posture changes of the patient.

Due to the aforementioned reasons, in the past years, we have witnessed a growing interest in robotic probes for soft tissue palpation in robot-assisted minimally invasive surgery (RMIS). Several types of rigid probe for stiff-inclusion identification in soft tissue with different types of tactile sensors situated at the tip have been proposed, such as the

The work described in this paper is supported in part by the U.K. Engineering and Physical Sciences Research Council under Grant EP/I028765/1.

N. Sornkarn and T Nanayakkara are with the Department of Informatics, Kings College London, Strand, London WC2R 2LS, UK (e-mail: nantachai.sornkarn@kcl.ac.uk, thrish.antha@kcl.ac.uk) optical tactile array probe [2], and force/stiffness-feedback probe [3], [4]. Given this direct measurement from the rigid probes, the capability of the sensing system is nevertheless constrained by its fixed morphology [5].

On the other hand, active sensing in humans does not solely involve the haptic feedback but also includes the modulation of proprioceptive feedback from spindles and tendons embedded in each muscle in order to enhance perception [6]. Previous studies suggest that humans use a variety of force/velocity control strategies during manual palpation [7], [8]. The strategies include the movement of finger in various trajectories, velocities, and frequencies; and the regulation of applied force [9]. The physical properties of the environment can be extracted by implicating appropriate strategy combinations, [10]-[12], i.e. surgeons can directly localize and extract the physical properties of the stiff-inclusions inside an artificial silicon phantom. There is evidence to suggest that the modulation of the internal state of the body and behavior accompany changes in the proprioceptive sensors [13]-[15].

Resently, we have shown in [16] that there is a relationship between the entropy of perception information and the internal impedance of the body. In this paper, we investigate the individual and collective role of internal impedance and behavioral variables in the accuracy of estimating an environmental variable using a controllable stiffness probe. We pose the hypothesis that a controllable stiffness robotic probe can use prior experience of its proprioceptive sensors in known environments to improve the accuracy of estimating an environmental variable (the depth of a buried nodule in a soft tissue in this case), by exploring both in behavioral (i.e. probing velocity and indentation) as well as internal impedance (stiffness of a Mckibben type joint in this case) spaces to maximize information gain in a Bayesian inferencing framework. We found that 1) The information gain (transfer entropy) in a Bayesian inferencing framework leads to a monotonic increase of estimation accuracy across trials irrespective of the sequence of recruiting different combinations of stiffness, indentation, and speed of the probe, 2) Exploration in morphological (stiffness) and behavioral (indentation and speed) space of the probe leads to better accuracy of estimating the depth of the nodule than a fixed combination across trials, 3) The internal stiffness of the soft probe plays a statistically significant role in the accuracy of nodule's depth estimation, 4) Information gain (transfer entropy) across trials and across morphological (stiffness) and behavioral (indentation and speed) combinations can be used to improve the efficiency of exploration. 


\section{EXPERIMENTAL SETUP}

\section{A. Design}

The design of the variable stiffness probe used in this experiment is developed from the probe's design in our previous studies. The probe, as shown in Figure 1 (a) comprises of two rigid links - tip link of length $l_{1}=80 \mathrm{~mm}$, and base link $l_{2}=70 \mathrm{~mm}$ made from ABS plastics. The joint coupled between these two links contains a variable stiffness mechanism with two linear ENTEX No.3552 stock springs from Advanex Europe Ltd. each with rating of $0.24 \mathrm{~N} / \mathrm{mm}$. Inside the base link (shown in Figure 1 (b)), there are two chambers dedicated for the linear springs which are connected between the pivot joint at the connecting point (the relative angle of the connecting point and the vertical axis of the tip link is zero) and the anchor ring via a microfilament thread. The stiffness of the joint can be mechanically controlled by changing the position of the anchor ring. The anchor ring is mounted on to the endeffector of a linear actuator L12-50-210-06-I from Firgelli Technologies Inc., which controls its position.

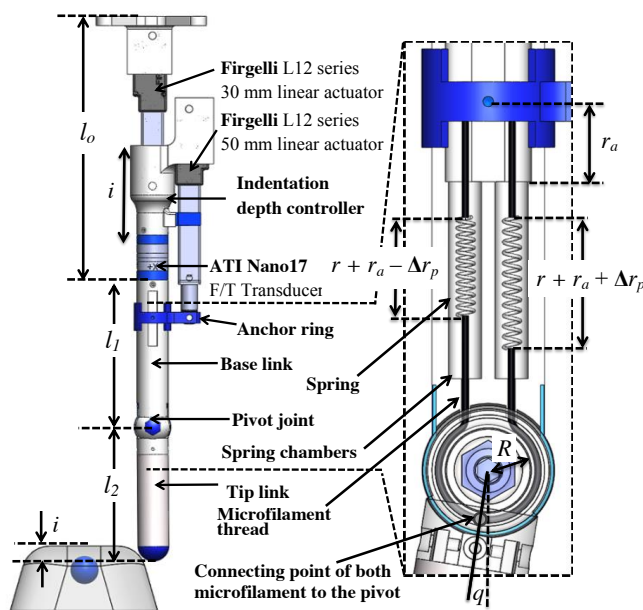

(a) (b)

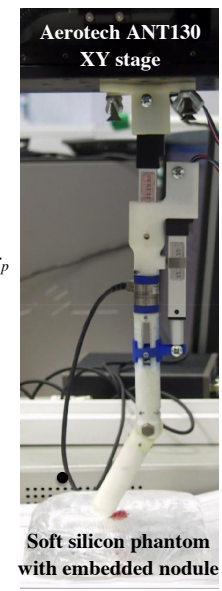

(c)

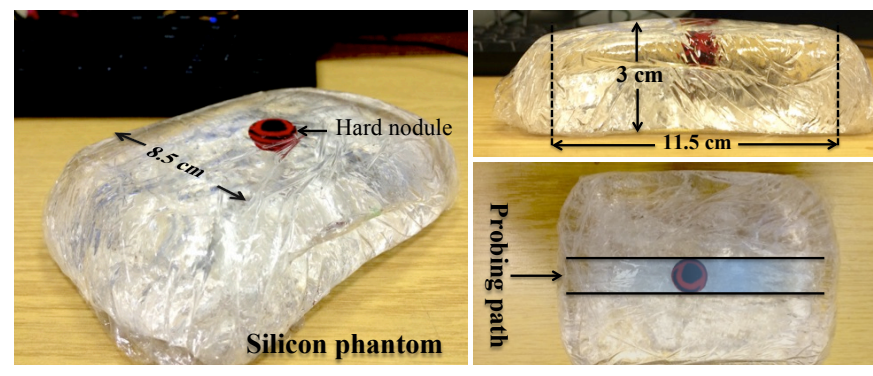

Fig. 1. (a) Design of this robotic probe comprises of two Firgelli L12 linear actuators to control the indentation and stiffness of the probe and the ATI Nano17 F/T transducer mounted at the top-end of the base link to measure the torque during the interaction with artificial soft tissue. (b) Two springs located inside the spring chambers are attached with the anchor ring and the pivot joint through a microfilament thread. (Note that the springs shown here are for illustrative purpose only) (c) Photo of the complete experimental platform's design comprising of the variable stiffness probe mounted on XY-stage. (d) A soft silicon phantom replicating a soft tissue with a spherical plastic bead of size $15 \mathrm{~mm}$ diameter embedded inside at different depths.
An ATI Nano17 6-axis force/torque transducer is mounted at the base of the probe to capture torque data during palpation. On top of the force sensor at the end of the base link, there is a connector to another $30 \mathrm{~mm}$-stroke-length linear actuator L12-30-100-06-I from Firgelli Technologies Inc. for controlling the indentation level, $i$. The length from the upper end of the base link to the mounting side of the probe is denoted by $l_{o}$ and has the initial value of $143 \mathrm{~mm}$. The total length of this probe when $q=0$ is $293 \mathrm{~mm}$. The probe structure is mounted on a flipped ANT130 XY-stage from Aerotech Inc. in the experimental setup as shown in Figure 1 (c), which allows the planar movement in $\mathrm{X}$ and $\mathrm{Y}$ direction of the probe.

In the experiment, we used soft silicon phantoms with an embedded hard nodule to replicate the soft tissue with stiff-inclusion. Silicon phantom is made from a soft clear silicon elastomer gel RTV27905 from Techsil. Three silicon phantoms were used as samples where the nodules are embedded at the depth of 2,4 , and $8 \mathrm{~mm}$ from the top surface of the phantom to the top of a nodule.

\section{B. Numerical analysis of variable joint stiffness mechanism}

From Figure 1(b), the rest length of both springs are denoted by $r$. The change of the length of both springs $\Delta r_{1}$, and $\Delta r_{2}$, are the result from the change in the displacement of anchor point, $r_{a}$, and the change in linear displacement $\Delta r_{p}$ due to the change in angular displacement of the probe, $q$, where $\Delta r_{p}=q R$. $R$ is the radius of the pivot joint at which the microfilament is attached to. Hence:

$$
\Delta r_{1}=r_{a}-q R \quad \text { and } \quad \Delta r_{2}=r_{a}+q R .
$$

Given that the identical springs are used inside both spring chambers with spring constant of $k_{s}$, the force contribution from each spring can be computed as follows:

$$
\overrightarrow{f_{s i}}=\Delta r_{i} k_{s}
$$

The torque provided from both springs due to the change of joint's angular displacement and the position of the anchor ring is:

$$
\tau_{s i}=\vec{f}_{s i} \times R=\vec{f}_{s i \perp} R,
$$

where $\vec{f}_{s i \perp}$ is the force perpendicular to the rotational axis; and $i=1$, and 2 .

$$
\vec{f}_{s i \perp}=f_{s i} \sin (q) .
$$

Therefore, the total torque developed due to both springs can be computed from Equation (2) to (4) as follows:

$$
\begin{aligned}
\tau_{s} & =\tau_{s 1}+\tau_{s 2} \\
& =R k_{s} \sin (q)\left(\Delta r_{1}+\Delta r_{2}\right)
\end{aligned}
$$

and the stiffness at the joint, $K_{s}$, is the derivatives of torque produced with respect to the angular displacement of the pivot joint, $q$, from Equation (5)

$$
K_{s}=\frac{\partial \tau_{s}}{\partial q}=2 r_{a} R k_{s} \cos (q) \text {. }
$$


The following figures depict simulated joint torque and joint's stiffness generated from following parameters: $r_{a}=$ $[0 \ldots 15] \mathrm{mm}, R=6.8 \mathrm{~mm}, q=[-90 \ldots 90]^{\circ}$, and $k_{s}=$ $0.24 \mathrm{~N} / \mathrm{mm}$.

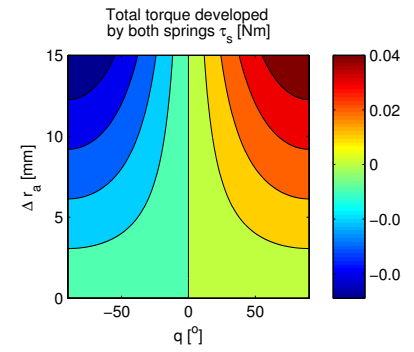

(a)

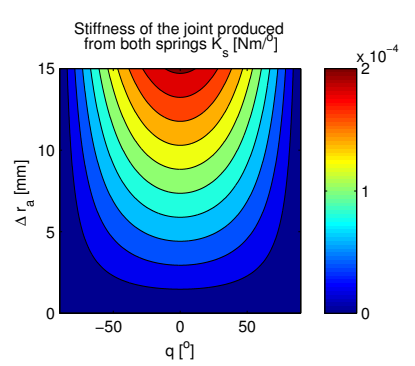

(b)
Fig. 2. Torque (a) and the stiffness (b) produced at the pivot joint due to the changes in the displacement of the anchor ring, $r_{a}$, and the angular displacement of the joint, $q$

Figure 2(a) illustrates the landscape of joint torque, $\tau_{s}$ due to the changes of both $q$ and $r_{a}$. It is shown here that the shape of the landscape representing the relationship between $\tau_{s}$ and $q$ depends on $r_{a}$. Taking the derivatives of this landscape of $\tau_{s}$ with respect to $q$ results in the stiffness profile, $K_{s}$, of the joint in relation to $r_{a}$ and $q$ as shown in Figure 2(b). The relationship between $K_{s}$ and $q$ becomes almost linear as the anchor ring approaches its origin at $r_{a}=0$. Since the profile of joint-angle-dependent stiffness, $K_{s}(q)$ can be controlled by changing $r_{a}$, for the rest of this paper, the joint stiffness level is determined by the position of the anchor ring, $r_{a}$.

\section{EXPERIMENTS AND RESULTS}

\section{A. Construction of Memory Primitives}

In this experiment, we explore whether a probe with controllable stiffness and variable probing behavior can exploit its past memory of palpation by varying its own internal stiffness and probing behavioral variables to maximize information gain or perceiving the depth of embedded nodule inside a soft tissue. Here we present the past memory of palpation in a form of memory primitives of the measured torque as a function of all varying behavioral and internal stiffness variables. In this section we illustrates how the experimental data are collected during the training phases and how these can be used to construct the probability distribution for different combinations of internal stiffness and behavioral variable.

The XY-Table is programmed to move the probe in a straight line along the probing path over the surface of the soft silicon phantom as shown in Figure 1 (d). The torque generated due to the interaction with soft tissue was measured at the rate of $1000 \mathrm{~Hz}$ around the $\mathrm{F} / \mathrm{T}$ transducer's $\mathrm{x}$-axis given different combinations of probe's joint stiffness, represented by $r_{a}$, probe's indentation $i$, probing velocity $v_{\text {probe }}$, and depth of nodule $d$, as shown in Table I. For each given combination, 25 palpation trials were repeated in order to generate the probability distribution of torque to construct
TABLE I

EXPERIMENTAL CONDITIONS

\begin{tabular}{|l|c|c|c|}
\hline Experimental variables & Sym. & Values & Units \\
\hline $\begin{array}{l}\text { Probe's stiffness } \\
\text { (anchor position) }\end{array}$ & $r_{a}$ & $\{0,4,8,12,16\}$ & $\mathrm{mm}$ \\
\hline $\begin{array}{l}\text { Relative distance between the } \\
\text { tip of the probe at rest and } \\
\text { the surface of tissue, i.e. } \\
\text { inwards tissue (indentation) }\end{array}$ & $i$ & $\{3,5,7,9,11\}$ & $\mathrm{mm}$ \\
\hline Probe's velocity & $v_{\text {probe }}$ & $\{10,20,30\}$ & $\mathrm{mm} / \mathrm{s}$ \\
\hline Nodule's depth & $d$ & $\{2,4,8\}$ & $\mathrm{mm}$ \\
\hline $\begin{array}{l}\text { Distance between the } \\
\text { XYplate and bottom of tissue }\end{array}$ & $l_{t}$ & 320 & $\mathrm{~mm}$ \\
\hline
\end{tabular}

the memory primitives, which can be used in the statistical approach described in the next section, in order to allow the estimation of the nodule's depth.

Each measured torque from the F/T transducer was denoised for 5 levels using wavelet decomposition technique with a Daubechie's db10 mother wavelet. From all 25 trials for each interaction condition, the probability distribution of torque, $P\left(\tau_{f} \mid d, i, r_{a}, v_{\text {probe }}\right)$, can be constructed under different nodule's depth, $d$, given different combination of probe's indentation, $i$, probe's stiffness, $r_{a}$, and probing velocity, $v_{\text {probe }}$ as shown in Table I. This results in total of 225 different interaction conditions. Here, only 81 interaction conditions are chosen to depict how the constructed memory primitives look like as shown in Figure 3 (a), (b), and (c).

\section{B. Experimental results and analysis}

1) Bayesian Inference in Estimating the Nodule's depth: In this section, we use Bayesian Inference approach in analysing the real-time torque data captured during the sweep of the probe over the area where a nodule is embedded inside an artificial tissue at different depths, in order to exploit the existing memory primitives to estimate the potential depth of the nodule. Here the iterated equation for Bayesian Inference is as following:

$$
P_{t}\left(d \mid \tau_{f}\right)=\frac{P\left(\tau_{f} \mid d, \gamma\right) P_{t-1}(d)}{\sum_{n=1}^{m} P\left(\tau_{f} \mid d_{n}, \gamma\right) P_{t-1}\left(d_{n}\right)},
$$

where $t$ is the current estimation iteration, $n$ is the index of $d$, and $m=3$ is the number of possible depth's estimation. $P_{t}\left(d \mid \tau_{f}\right)$ represents the posterior probability distribution of nodule's depth given the measured torque, $\tau_{f}$ computed from the prior distribution $P_{t-1}(d)$ and the sampling or likelihood probability distribution of torque, $P\left(\tau_{f} \mid d, \gamma\right)$ given depths and different set of internal stiffness variable and probing behaviors, $\gamma \in\left\{r_{a}, i, v_{\text {probe }}\right\}$ presented in the memory primitives, $\varphi$. The posterior computed at each trial or iteration is then used to update the probability distribution of the depth as a prior distribution in the next iteration. The initial prior of the function $P_{t=0}(d)$ has a flat distribution across different depths, reflecting the equal probability.

Here, we assess the performance of using Bayesian Inference to estimate the nodule's depth across iterations, given different interaction conditions, $\gamma$, shown in Table I. The procedure for the assessment can be found in Algorithm 1. 


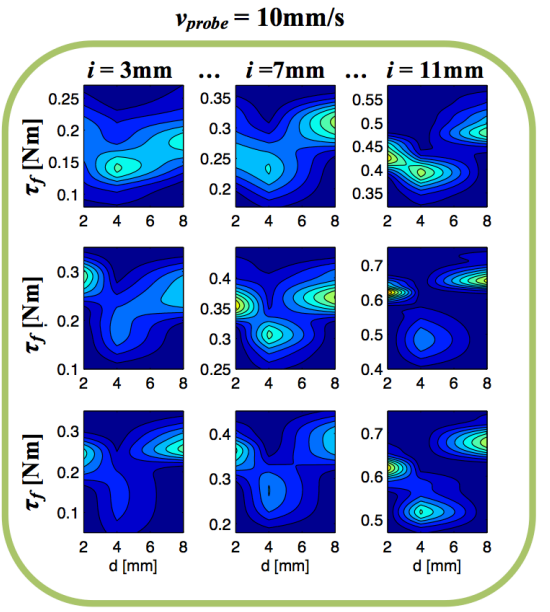

(a)

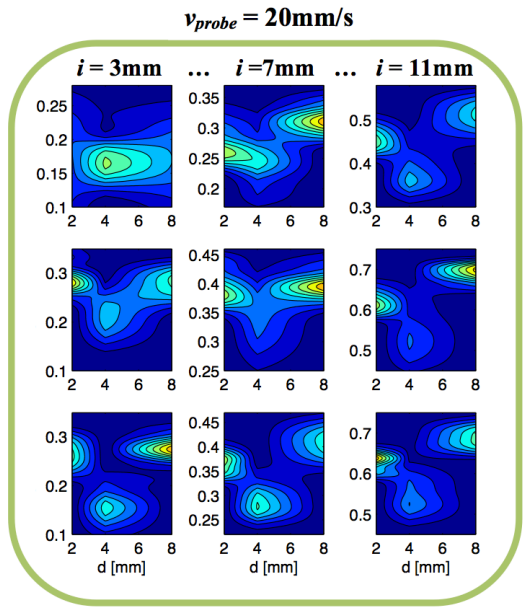

(b)

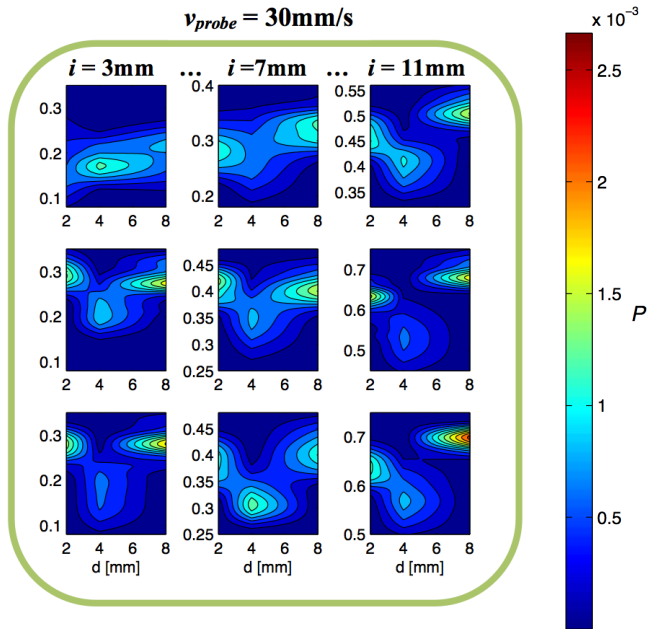

(c)

Fig. 3. Examples of memory primitives computed as probability function of the de-noised torque profiles from 25 trials given different interaction conditions shown in Table I. The sample of memory primitives shown here consist of those when the probing velocity, $v_{p}$ robe $=10$, 20, and 30 , in subfigures (a,b,c-1), (a,b,c-2), and (a,b,c-3), for the indentation level, $i$, of 3,7 , and $11 \mathrm{~mm}$, and the stiffness of the joint denoted by $r_{a}$, of 0,4 , and $16 \mathrm{~mm}$.

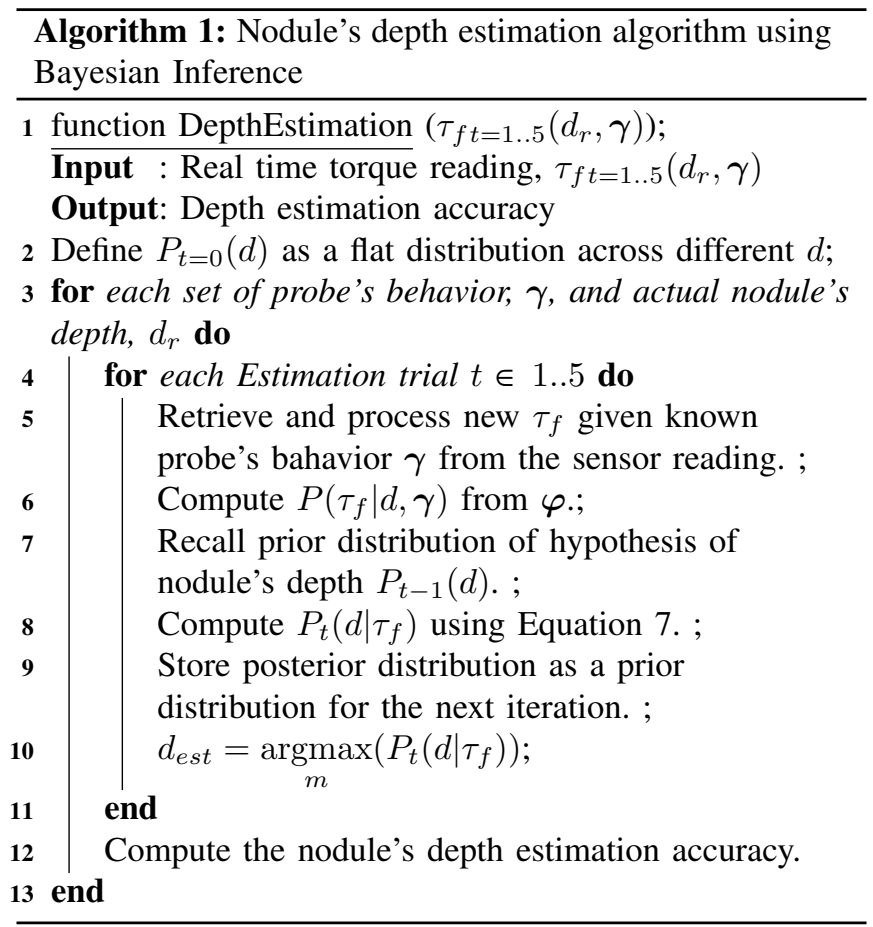

Results of using 5-iterations Bayesian Inference to estimate the depth of the nodule given different interaction conditions are shown in Figure 4. In Figure 4, each subplot contains the mean and standard errors of estimated depth across all probe's indentation from first to fifth Bayesian inference iterations for $v_{\text {probe }}=10,20$, and $30 \mathrm{~mm} / \mathrm{s}$ are shown in red, green, and blue curves respectively for a given probe's stiffness, $r_{a}$. Each column and row represents the given probe's stiffness, $r_{a}$, and the actual nodule's depth respectively.

The accuracy in depth estimation is shown in Figure
5. The estimation accuracy increases to around $84 \%$ as the number of Bayesian inference iteration increases from 1 to 5. From Figure 5, we can see that the estimation accuracy is higher when the nodule is buried closer to the tissue's surface. At the final iteration, the estimation accuracy when the actual nodule's depth, $d_{r}=2,4$, and $8 \mathrm{~mm}$ are approximately $85.3 \%, 84 \%$, and $82.7 \%$ respectively. The estimation accuracy for all depth ranges tends to increase as the number of iteration increases. However, higher number of iteration would average out the data which mostly represent in the test sample itself. Hence, the number of iteration is limited to maximum of 5 , in this case.

Additionally, as shown in Figure 4, the convergence rate of the distribution of the nodule's depth estimation is different for different interaction conditions. Some converges at faster rate than the other. This leads to the question as to how we can determine or quantify the sufficiency of the number of iterations or explorations required to make an estimation about the depth. This problem can be addressed by the measurement of transfer entropy based on the information gain metrics, which is explained in Subsection III-B.2.

2) Kullback-Liebler Transfer Entropy: Information transfer entropy can be used to observe the directed information exchanges between two systems/variables, which quantifies the common influences of two coupled systems/factors [17]. In other words, mutual information between probing behavior (random variable $\mathrm{A}(\mathrm{RV}-\mathrm{A})$ ) and torque sensor reading (random variable $B$ (RV-B)) doesn't change with the exchange of variables, whereas, the transfer entropy from $\mathrm{RV}-\mathrm{A}$ to RV-B is different from the transfer entropy from RV-B to RV-A. Kullback-Liebler (KL) divergence quantifies this transfer entropy. In this context, KL-divergence can be used to determine whether the nodule's depth estimation procedure require any further measurements to make an accurate estimation.

If we consider a set of $P_{t}\left(d \mid \tau_{f}\right)$ as the hypothesis of the 

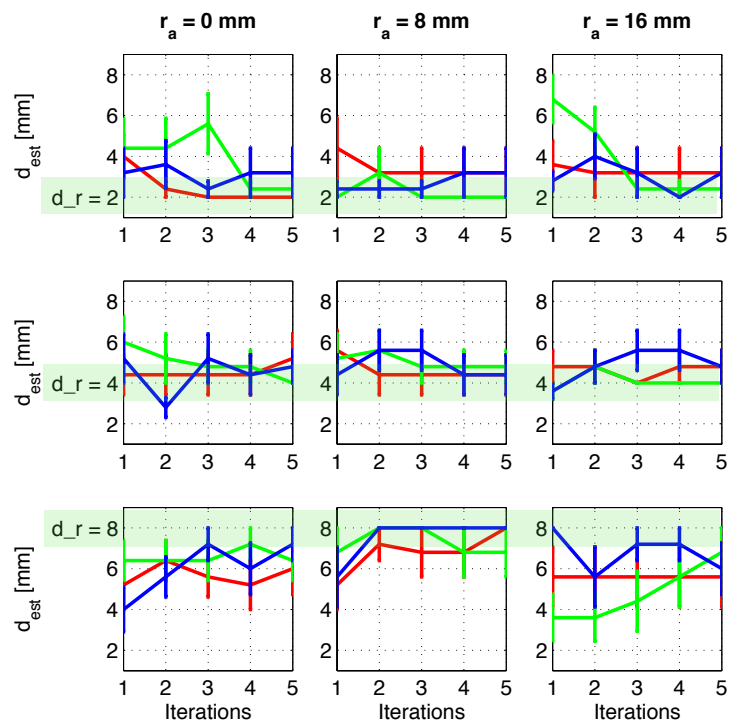

$v_{\text {probe }}=10 \mathrm{~mm} / \mathrm{s}$

actual nodule's depth $d_{r}$

Fig. 4. Examples of mean and standard errors of estimated depth across all probe's indentation from first to fifth Bayesian inference iterations for $v_{\text {probe }}=10,20$, and $30 \mathrm{~mm} / \mathrm{s}$ are shown in red, green, and blue curves respectively for a given probe's stiffness, $r_{a}$. The real depth of nodule, $d_{r}$, assessed here are from those existed in the memory primitives explained earlier, namely: $d_{r}=2,4$, and $8 \mathrm{~mm} . d_{r}$ : Actual depth of the nodule, $r_{a}$ : represents stiffness of the probe, $i$ : probe's indent, and $v_{\text {probe }}$ : palpation velocity. Here in the plots, the actual depths are highlighted in green.

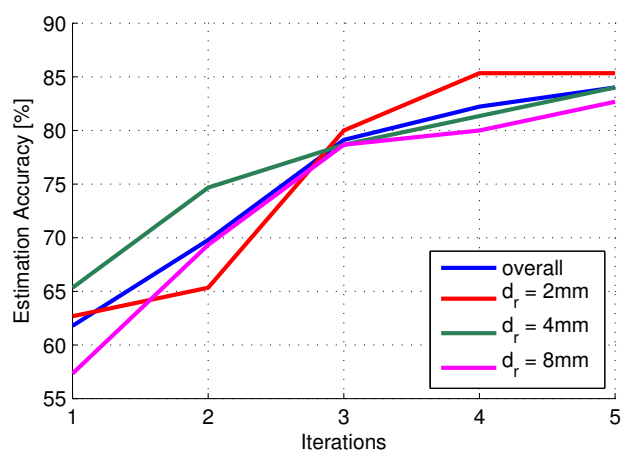

Fig. 5. The resulting overall nodule's depth estimation accuracy shown in blue line depicts that the estimation accuracy reaches $84 \%$ after 5 iterations. The estimation accuracy for each individual actual depth, $d_{r}=2$, 4, and $8 \mathrm{~mm}$ are represented by red, green and magenta lines respectively.

depth estimation, its entropy for a given torque measurement, $\tau_{f}$, is dependent on a set of probe's stiffness and probing behavior, $\gamma$, i.e. $r_{a}, i$, and $v_{\text {probe }}$. KL-divergence defined in equation (8) represents the additional information gained, $G$, about the relationship between the hypothesis of depth estimation, $P_{t}(d)$, and $\tau_{f}$ across iterations of Bayesian Inference as well as across different sets the probe's stiffness and palpation's behavior. Therefore, KL-divergence is a good measure to quantify the gain of different actions underlying the changes in the behavior.

$$
G_{t}=P_{t}\left(d \mid \tau_{f}\right) \log \frac{P_{t}\left(d \mid \tau_{f}\right)}{P_{t=0}(d)}
$$

$P_{t}\left(d \mid \tau_{f}\right)$ represents the probability distribution of depth estimation obtained from Equation (7) at $t^{t h}$ iteration, and $P_{t=0}(d)$ represents the base hypothesis about the nodule's depth estimation.

KL-divergence is implemented in addition to the Bayesian Inference method to determine the number of measurement required to estimate the nodule's depth by computing the correlation distance, $\delta$, between information gain of the current hypothesis, $G_{t}$, and that of the prior hypothesis, $G_{t-1}$, in relation to the base prior distribution, $P_{t=0}(d)$. The palpation process stops at the point where the correlation distance is less than empirically specified threshold, $T=$ 0.0005 , signifying that there is none to little change in the information gained across iterations. The depth estimation procedure is shown in Algorithm 2.

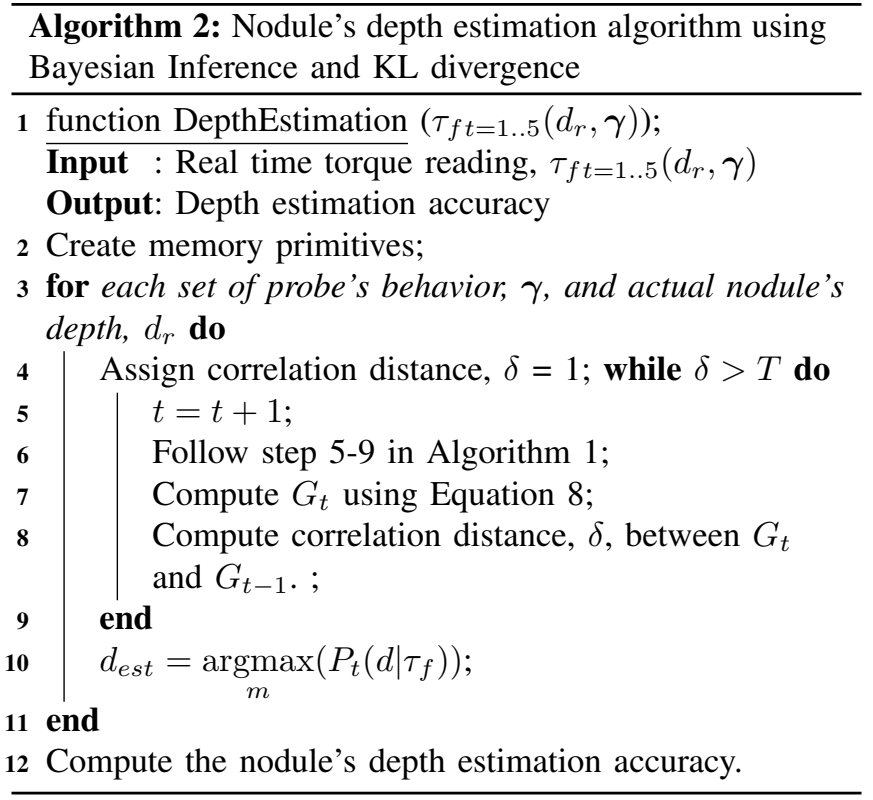

Through the implementation of KL-divergence, the nodule's depth estimation procedure requires on average of only 3.8 iterations with standard deviation of 1.2 iterations. Nonetheless, the overall depth estimation accuracy is approximately $84 \%$ as shown in Figure 6 (orange bars), comparable to the process with fixed 5- iterations. And the accuracy of nodule's depth estimation for each actual depth are approximately $87 \%, 85 \%$, and $80 \%$ for $d_{r}=2,4$, and $8 \mathrm{~mm}$ respectively. From the results, it can be interpreted that applying this method allows the procedure to dynamically minimize the number of exploration that would be sufficient to make an estimation about the depth of the nodule with comparable performance to those with static 5-iterations.

Up to this point, the nodule's depth estimation procedure is constrained by the exploitation of a single memory primitives across iterations for each exploration, i.e. no change in probe's stiffness and probing behavior across iterations. Next, 
we explore whether the accuracy of nodule's depth estimation can be enhanced by the modulation of combination of probe's stiffness and probing behavioral variables, $\gamma$. That is to allow the estimation procedure to explore multiple memory primitives. In order to assess this, we perform a similar procedure to that shown in Algorithm 2, but instead of a set of pre-defined probing behaviors, the probe's stiffness and probing behavioral variables are randomly selected across iterations. This process is repeated for 100 trials for each artificial soft tissues, in which the nodule is embedded at $d_{r}$ $=2,4$, and $8 \mathrm{~mm}$.

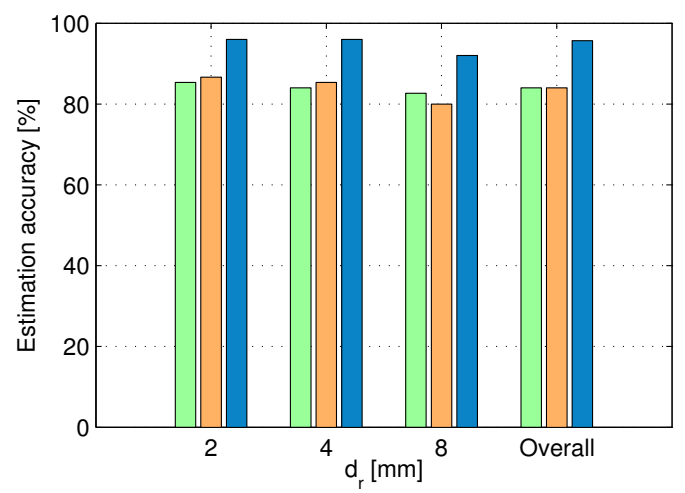

5-iteration Baysian Inference (with fixed stiffness and probing behavior across trials) Bayesian Inference with $\mathrm{KL}$-divergence (with fixed stiffness and probing behavior across trials) Bayesian Inference with $\mathrm{KL}$-divergence (with random stiffness and probing behavior across trials)

Fig. 6. Overall nodule's depth estimation accuracy when using different approaches: 1) 5-iteration Bayesian inference without KL-divergence (shown in green), 2) the Bayesian Inference together with the KL-Transfer Entropy with fixed probe's stiffness and probing behavioral variables (shown in orange), and 3) the Bayesian Inference together with the KL-Transfer Entropy with random probe's stiffness and probing behavioral variables (shown in blue).

The overall average accuracy from 100 trials of nodule's depth estimation using Bayesian Inference with KL-Transfer Entropy with random probing behavior across iterations reaches almost $95 \%$ as shown in Figure 6 in pale blue bar. The estimation accuracy from all individual actual depths are also higher in comparison to those with pre-defined $\gamma$. Furthermore, this also results in on average slightly lower number of Bayesian Inference iteration, with the average of 3.5 iterations with standard deviation of 1.2 iterations, required to gain sufficient information for making an estimation.

All in all, the implementation of information gain metrics allows the information across each iteration of Bayesian Inference to be quantified. The results from both depth estimation procedures suggest that this allows the estimation process to stop when there is sufficient information for nodule's depth estimation, i.e. no new information is gained by taking any further action. The first analysis of this subsection shows that it is not necessary, in some cases, to perform up to 5 iterations to obtain the equivalent estimation accuracy to the previous section shown in Figure 5. Furthermore, by allowing the probing behavior to randomly modulate across iterations, the average depth estimation accuracy increases to almost $95 \%$. Being able to change $\gamma$ across iteration allows the exploration of multiple memory primitives, which can lead to global optimum.

\section{Discussion AND CONCLUSION}

This paper has explored the individual and collective role of internal stiffness of the probe and probing behavioral variables in the accuracy in estimating an environmental variable using a controllable stiffness probe. Firstly, we designed and fabricated a two link robotic probe with a controllable stiffness joint and a mechanism to control the indentation level. Then, we posed the problem of using only torque data measured real-time during a palpation trial over an artificial soft tissue with a nodule embedded inside to estimate the depth of the nodule under different probing behaviors. We conducted experiments across 3 levels of probing velocity, 5 levels of indentation, and 5 levels of joint stiffness, for 3 depths of the nodule, with 25 trials per combination. In total 5625 probing trials were performed using an automated experimental setup.

Our experimental results show that the information gain under Bayesian inferencing framework leads to improvement in the accuracy of estimating the environmental parameter (in this case the depth of the buried nodule) irrespective of how the probing behavior and internal stiffness of the probe are controlled across trials. However, the results show that not all combinations of probing behaviors and probe stiffnesses render the same accuracy of estimating all the depths of the nodule. This informs the practice of manual and robotic probing behaviors as well as providing useful design guidelines for soft robotic probes with controllable stiffness. In addition, the exploration in probe's stiffness and probing behavioral (indentation and probing velocity) spaces results in higher nodule's depth estimation accuracy. Information gain (transfer entropy) across trials and across morphological (stiffness) and behavioral (indentation and speed) combinations can be used to improve the efficiency of exploration. The experimental results also show that the stiffness of the embodiment plays a statistically significant role in embodied perception.

In biological counterpart like in human manual palpation, we witnessed different probing behaviors such as the vibration, sliding, and changes in various behavioral variables. Based on the experimental results presented in this paper, it is fair to predict that the reasons behind those behaviors are the exploration in the memory primitives collected from past experience across different morphological and behavioral spaces to explore the properties of diagnosed soft-tissues. Human may also employ the combinations of statistical strategies similar to those proposed in this paper in making accurate estimation.

This paper has provided important guidelines to design variable behavior probe inclusive of stiffness and indentation level regulation function and the construction of internal 
memory primitives to estimate the depth of the nodule using Bayesian Inference together with information gain metrics. Certainly, this probe used in the experiment cannot yet be realized in the real operation scenario as in palpation, there are many other varying critical factors such as the shape and roughness of the surface, the friction of the surface, stiffness of the tissues, and etc. Nonetheless, this paper underlies the important perspective that the internal stiffness of the body as well as the behavior of an agent can influence how the agent perceive the environment. These findings contribute to our understanding in biological active perception or active sensing, where an action is required to accurately perceive the environment, because the perception and action are mediated by a shared embodiment. Therefore, it is important to note that biological haptic perception does not only depend on the environmental conditions, but also on the behavioral state of the agents. In the next stage, it would be interesting to implement reinforcement learning algorithm in such system to allow not only on-line nodule's depth estimation but also real-time learning and enriching of the memory primitives.

\section{REFERENCES}

[1] O. Van der Meijden and M. Schijven, "The value of haptic feedback in conventional and robot-assisted minimal invasive surgery and virtual reality training: a current review," Surgical endoscopy, vol. 23, no. 6, pp. 1180-1190, 2009.

[2] H. Xie, H. Liu, S. Luo, L. Seneviratne, and K. Althoefer, "Fiber optics tactile array probe for tissue palpation during minimally invasive surgery," in Intelligent Robots and Systems (IROS), 2013 IEEE/RSJ International Conference on, Nov 2013, pp. 2539-2544.

[3] H. Liu, J. Li, X. Song, L. Seneviratne, and K. Althoefer, "Rolling indentation probe for tissue abnormality identification during minimally invasive surgery," Robotics, IEEE Transactions on, vol. 27, no. 3, pp. 450-460, June 2011.

[4] B. Ahn, Y. Kim, and J. Kim, "New approach for abnormal tissue localization with robotic palpation and mechanical property characterization," in Intelligent Robots and Systems (IROS), 2011 IEEE/RSJ International Conference on, Sept 2011, pp. 4516-4521.

[5] K. Sangpradit, H. Liu, P. Dasgupta, K. Althoefer, and L. Seneviratne, "Finite-element modeling of soft tissue rolling indentation," Biomedical Engineering, IEEE Transactions on, vol. 58, no. 12, pp. 33193327, Dec 2011.

[6] A. Simpkins, "Robotic tactile sensing: Technologies and system (dahiya, r.s. and valle, m.; 2013) [on the shelf]," Robotics Automation Magazine, IEEE, vol. 20, no. 2, pp. 107-107, June 2013.

[7] J. Konstantinova, M. Li, G. Mehra, P. Dasgupta, K. Althoefer, and T. Nanayakkara, "Behavioral characteristics of manual palpation to localize hard nodules in soft tissues," Biomedical Engineering, IEEE Transactions on, vol. PP, no. 99, pp. 1-1, 2014.

[8] N. Wang, G. J. Gerling, R. M. Childress, and M. L. Martin, "Quantifying Palpation Techniques in Relation to Performance in a Clinical Prostate Exam," IEEE Trans. on Information Technology in Biomedicine: a Publication of the IEEE Engineering in Medicine and Biology Society, vol. 14, no. 4, pp. 1088-97, Jul. 2010. [Online]. Available: http://www.ncbi.nlm.nih.gov/pubmed/20172838

[9] J. Morley, A. Goodwin, and I. Darian-Smith, "Tactile discrimination of gratings," Experimental Brain Research, vol. 49, no. 2, pp. 291-299, 1983. [Online]. Available: http://dx.doi.org/10.1007/BF00238588

[10] L. A. Jones and S. J. Lederman, Human hand function. Oxford University Press, 2006.

[11] G. Robles-De-La-Torre and V. Hayward, "Force can overcome object geometry in the perception of shape through active touch," Nature, vol. 412, no. 6845, pp. 445-448, 07 2001. [Online]. Available: http://dx.doi.org/10.1038/35086588

[12] S. J. Lederman and R. L. Klatzky, "Extracting object properties through haptic exploration," Acta Psychologica, vol. 84, no. 1, pp. 29 - 40, 1993, tactile Pattern Recognition. [Online]. Available: http://www.sciencedirect.com/science/article/pii/0001691893900708
[13] T. J. Prescott, M. E. Diamond, and A. M. Wing, "Active touch sensing," Philosophical Transactions of the Royal Society of London B: Biological Sciences, vol. 366, no. 1581, pp. 2989-2995, 2011.

[14] R. Pfeifer, E. A1, R. Pfeifer, M. Lungarella, and F. Iida, "Selforganization, embodiment, and biologically inspired robotics," Science, vol. 318, 2007.

[15] M. Hoffmann and R. Pfeifer, "The implications of embodiment for behavior and cognition: animal and robotic case studies," CoRR, vol. abs/1202.0440, 2012.

[16] N. Sornkarn, M. Howard, and T. Nanayakkara, "Internal impedance control helps information gain in embodied perception," in Robotics and Automation (ICRA), 2014 IEEE International Conference on, May 2014, pp. 6685-6690.

[17] T. Schreiber, "Measuring information transfer," Phys. Rev. Lett., vol. 85, pp. 461-464, Jul 2000. [Online]. Available: http://link.aps.org/doi/10.1103/PhysRevLett.85.461 